\title{
Jubilación, trastornos psicofisiológicos y bienestar psicológico en una muestra de adultos mayores argentinos
}

\section{Retirement, psychophysiological disorders and psychological well-being in a sample of Argentinian older adults}

\author{
Paula Daniela Hermida ${ }^{1}$ \\ María Florencia Tartaglini ${ }^{2}$ \\ Carolina Feldberg ${ }^{3}$ \\ Dorina Stefani ${ }^{4}$
}

${ }^{1,4}$ Instituto de Investigaciones Cardiológicas, Universidad de Buenos Aires. Argentina

${ }^{2}$ Instituto de Neurociencias Buenos Aires. Argentina

${ }^{3}$ Servicio de Neurología Cognitiva, Neuropsicología y Neuropsiquiatría, Fundación para la Lucha contra las Enfermedades Neurológicas de la Infancia. Argentina

\section{1,2, 3,4 Consejo Nacional de Investigaciones Científicas y Técnicas. Argentina}

\begin{abstract}
Resumen: El objetivo de este estudio fue describir la relación entre trastornos psicofisiológicos y bienestar psicológico en la jubilación, en un grupo de adultos mayores argentinos. Se utilizó un diseño de estudio "ex post facto", de carácter retrospectivo, simple. 150 adultos mayores (autoválidos, de nivel socioeconómico medio y diferente género, residentes en Buenos Aires y zonas de influencia -Argentina-, y que poseían un vínculo laboral estable y formal antes de jubilarse) respondieron un Cuestionario de datos sociodemográficos y de perfil jubilatorio, un Cuestionario de estado de salud y el Índice de Bienestar Psicológico. Los resultados mostraron un mayor bienestar psicológico entre quienes informaron una menor presencia de trastornos psicofisiológicos asociados a la jubilación. Dichos hallazgos coincidieron con los de investigaciones previas destacando la importancia del estudio del bienestar psicológico como un aspecto central en la calidad de vida de los adultos mayores.
\end{abstract}

Palabras clave: adultos mayores, jubilación, bienestar psicológico, trastornos psicofisiológicos, envejecimiento

\begin{abstract}
The purpose of this study was to describe the relationship between psychophysiological disorders and psychological well-being during retirement in a group of Argentinian older adults. An ex post facto simple retrospective design was used. 150 older adults (self-sufficient, mixed-gender, of middle socioeconomic status, with stable and formal employment prior to retirement) answered a Socio-Demographic and Retirement Profile Background Questionnaire, a Health Status Questionnaire and the Psychological General Well-Being Index. The results show greater psychological well-being amongst those who presented less psychophysiological disorders related to retirement. These findings coincide with previous research and highlight the importance of studies on psychological well-being as a central aspect in older adult quality of life.
\end{abstract}

Key Words: older adults, retirement, psychological well-being, psychophysiological disorders, aging

Cómo citar este artículo:

Hermida, P. D., Tartaglini, M. F., Feldberg, C., \& Stefani, D. (2017). Jubilación, trastornos psicofisiológicos y bienestar psicológico en una muestra de adultos mayores argentinos. Ciencias Psicológicas, 11(2), 213 221. doi: https://doi.org/10.22235/cp.v11i2.1498

\footnotetext{
La infraestructura, el material y equipamiento necesario para el desarrollo del presente estudio fue provisto por el Instituto de Investigaciones Cardiológicas, ININCA-UBA-CONICET. Asimismo, el presente estudio fue financiado por el Consejo Nacional de Investigaciones Científicas y Técnicas (CONICET) a través del Proyecto de Investigación Plurianual "Estrés y envejecimiento masculino. Las creencias acerca del sentido de la vida y su relación con el envejecimiento satisfactorio" PIP No 112/20110100295 (2012-2014), bajo la dirección de la Dra. Dorina Stefani.
}

Correspondencia: Paula Daniela Hermida. Instituto de Investigaciones Cardiológicas, UBA-Marcelo T. de Alvear 2270, C1122AAJ, Ciudad Autónoma de Buenos Aires, Argentina, e-mail: paulahermida@conicet.gov.ar. María Florencia Tartaglini, e-mail: florenciatartaglini@ conicet.gov.ar; Carolina Feldberg, e-mail: cfeldberg@hotmail.com; Dorina Stefani, e-mail: dstefani@fmed.uba.ar; CONICET. 


\section{Introducción}

La jubilación es el suceso vital por medio del cual los sujetos dejan el trabajo. Se debe contemplar que el trabajar implica realizar una actividad en un determinado contexto, lo cual posee diferentes significados para el sujeto, a la vez que constituye un fenómeno social donde estructura su identidad en el vínculo con otros individuos (Dessors \& Guiho-Bailly, 1998; Peiró \& Prieto, 1996; Schvarstein \& Leopold, 2005). Así, la pérdida del trabajo a través de la jubilación implica el abandono del "rol" laboral desarrollado a lo largo de la vida adulta, y la necesidad de construcción de un nuevo "rol" (Biancotti et al., 2001; Téllez Rivera \& Reyes Montoya, 2004).

Asimismo, en las sociedades occidentales se considera, de forma arbitraria, que entre los $60 \mathrm{y}$ 65 años, edad en la que se deja de trabajar a través de la jubilación, se inicia la etapa de la vejez. Al respecto, se han ido generando diversas concepciones acerca de esta etapa vital. Mientras que a principios de siglo XX predominaba un modelo deficitario, que consideraba a la vejez como una etapa asociada a la enfermedad, fealdad y muerte, en la actualidad se sostiene un modelo más vinculado al desarrollo (Stefani, 2000). Al respecto Baltes, Reese y Lipssit (1980) afirman que en el transcurso de la vida el desarrollo es constante e intervienen diferentes variables: las normativas (relacionadas con los factores biológicos y sociales propios de la edad del sujeto), las contextuales (vinculadas al momento sociocultural en el que se encuentra inmerso), y las no normativas (vinculadas con su propia historia o recorrido, es decir las variables psicológicas). Así, se considera que en la vejez intervienen variables motoras, cognitivas y emocionales, pero también existen diferencias psicológicas interindividuales, atribuibles a la edad $\mathrm{y}$ en relación al contexto que rodea al individuo (Birren, 1996; Fernandez-Ballesteros 2007).

En esta línea, uno de los factores que incide de forma positiva o negativa en la adaptación a la vejez es el bienestar psicológico (Lawton, 1991). Dicho concepto fue abordado por diferentes autores, generándose una multiplicidad de definiciones. En todas ellas se observa la existencia de dos grandes componentes: uno emocional o afectivo, vinculado con los sentimientos de placer y displacer que experimenta la persona, y otro cognitivo, relativo a los juicios de valor que realiza el sujeto sobre lo vivido (Villar, Triadó, Solé Resano, \& Osuna, 2003).
Por su parte, Neugarten, Havighurst y Tobin (1961) lo definen como la sensación de bienestar derivada del grado de satisfacción que el adulto mayor posee acerca de si mismo y de lo vivido. Así, de acuerdo a los autores, la satisfacción vital, como constructo que refleja el bienestar psicológico del individuo y permite evaluarlo, se encuentra conformada por cinco aspectos: "entusiasmo por la vida", "resolución y fortaleza", "congruencia entre logros y aspiraciones", "autoestima positiva" y "tono emocional optimista". Bajo este marco teórico desarrollaron el Índice de Satisfacción Vital-A (Neugarten, Havighurst, \& Tobin, 1961). En estudios posteriores, tales como el de Zegers Prado, Rojas-Barahona y Förster Marín (2009), se observó que la dimensión de "resolución y fortaleza" no era correctamente evaluada por el instrumento, así como tampoco la de "autoestima positiva". Es por este motivo que frecuentemente, para evaluar el fenómeno de satisfacción vital, se utiliza también la Escala de Autoestima de Rosenberg (Rojas-Barahona, Zegers, \& Föster, 2008; Stefani, 2002). Dicho concepto es definido por Rosenberg (1965) como el sentimiento hacia uno mismo, de carácter negativo o positivo, resultante de una evaluación acerca de las características propias.

En los últimos años el estudio de bienestar psicológico en adultos mayores, a través del concepto de satisfacción vital, ha cobrado relevancia por ser considerado un indicador sanitario, desde una mirada evolucionada que integra diferentes aspectos de la salud y la enfermedad (CastilloCarniglia, Albala, Dangour, \& Uauy, 2012). Al respecto, Mroczek y Kolarz (1998) habían observado que la medida global de bienestar es estable a lo largo del tiempo, siendo un indicador de interés al no presentar modificaciones sustanciales vinculadas a los cambios asociados a la edad. Esto no implica que todos los adultos mayores posean altos niveles de bienestar psicológico, ya que ello dependerá de diversas variables o factores. En este sentido, Maddox (1964) ya sostenía que la actividad o participación social condiciona el bienestar psicológico.

Por otra parte, se ha observado que los individuos que presentan un mayor bienestar psicológico se adaptan mejor y afrontan de forma más exitosa la tensión generada por distintos estresores (Figueroa, Contini, Lacunza, Levín, \& Estévez, 2005; Jiménez, Izal, \& Montorio, 2016). El estrés psicológico, es un proceso en el que el individuo, al ex- 
perimentar cambios en su vida, podría evaluarlos como amenazantes, si considerara que exceden a su capacidad para hacer frente a la nueva situación (Lazarus \& Folkman, 1984). Se ha demostrado a su vez, que las situaciones estresantes desencadenan una respuesta fisiológica (Cano Vindel \& Miguel Tobal, 2000; Casado Morales, 1996). Desde esta perspectiva, el concepto de trastorno psicofisiológico incluye aquellas alteraciones físicas que son provocadas, agravadas o perpetuadas por factores psicosociales (Cano Vindel \& Miguel Tobal, 2000; Gatchel \& Bianchard 1993). En esta línea, se observó que situaciones sociales percibidas como estresantes pueden generar emociones negativas $\mathrm{y}$, como consecuencia, la aparición o empeoramiento de distintas alteraciones, tales como las respiratorias, cardiovasculares o inmunológicas (Cockerham, 2013; Piqueras Rodríguez, Ramos Linares, Martínez González, \& Oblitas Guadalupe, 2009; Wheaton, 1996). Al respecto, diversas investigaciones dieron cuenta de que la jubilación es uno de los principales eventos vitales estresantes que puede generar cambios importantes en la salud de las personas (Behncke, 2012; Holmes \& Rahe,1967; Jafri et al., 2017 ).

De esta forma, el presente trabajo se propone describir, en un grupo de adultos mayores jubilados argentinos, la presencia de trastornos psicofisiológicos vinculados a la jubilación y los niveles de bienestar psicológico, y analizar la posible asociación entre ambas variables.

\section{Materiales y Métodos}

\section{Diseño de investigación}

Se siguieron los pasos correspondientes al estudio "ex post facto", de carácter retrospectivo, simple (Montero \& León, 2002).

\section{Participantes}

La estrategia de muestreo utilizada fue la no probabilística, de tipo intencional, aplicada a los adultos mayores que asistían a centros de día y talleres dependientes del Gobierno de la Ciudad Autónoma de Buenos Aires y conurbano bonaerense (República Argentina). Se utilizó también el efecto de bola de nieve.

La muestra estuvo conformada por 150 adultos mayores autoválidos, de diferente género, mayores de 60 años, de nivel socioeconómico me- dio, residentes en un gran centro urbano (Ciudad Autónoma de Buenos Aires y conurbano, República Argentina) y que poseían un vínculo laboral estable y formal antes de jubilarse.

En la tabla 1 se presentan las medias aritméticas, desvíos estándar y recuentos de frecuencias y porcentajes, de acuerdo al nivel de medición, de las características del perfil sociodemográfico de la muestra total. De acuerdo a la versión abreviada del Índice de Nivel Económico Social de Gino Germani (Grimson et. al., 1972), en las respuestas consignadas por los adultos mayores sobre educación y ocupación se observa que todos los sujetos que conformaron la muestra pertenecen al nivel socioeconómico medio.

En cuanto al perfil jubilatorio de la muestra, presenta una edad promedio de jubilación de 65.29 años ( $d e=4.86$ años) y un tiempo promedio de estar jubilados de 9.31 años $(d e=5.57)$. En cuanto a la ocupación previa a la jubilación, expresan el ser "empleado" (26\%) "profesional independiente/ empresario" (22\%) o haber desarrollado "oficios varios" (23\%), y haber desarrollado dichos trabajos por "más de 15 años" (91\%).

Tabla 1.

Perfil sociodemográfico de la muestra total $(n=150)$

\begin{tabular}{|c|c|c|c|c|c|}
\hline$x$ & $\mathbf{n}$ & $\%$ & $x$ & $\mathbf{n}$ & $\%$ \\
\hline \multicolumn{3}{|l|}{ Sexo } & \multicolumn{3}{|l|}{ Practicante } \\
\hline Masculino & 86 & 57 & No & 93 & 62 \\
\hline Femenino & 64 & 43 & Si & 57 & 38 \\
\hline \multicolumn{6}{|l|}{ Nacionalidad } \\
\hline Argentino & 137 & 91 & Educación & & \\
\hline Extranjero & 13 & 9 & Primario & 40 & 26 \\
\hline \multicolumn{3}{|l|}{ Estado Civil } & Secundario & 46 & 31 \\
\hline Soltero & 22 & 15 & \multirow[t]{2}{*}{ Terciario/Universitario } & \multirow[t]{2}{*}{64} & \multirow[t]{2}{*}{43} \\
\hline Viudo & 38 & 25 & & & \\
\hline Divorciado o separado & 27 & 18 & Convive & & \\
\hline Casado o en pareja & 63 & 42 & No & 55 & 37 \\
\hline \multicolumn{3}{|l|}{ Tiene hijos } & Con pareja & 53 & 35 \\
\hline No & 42 & 28 & Con pareja e hijos & 13 & 9 \\
\hline $\mathrm{Si}$ & 108 & 72 & Con hijos & 16 & 10 \\
\hline & & & Otros & 13 & 9 \\
\hline \multirow{5}{*}{ Religión } & 27 & 18 & & & \\
\hline & 99 & 66 & Dónde Vive & & \\
\hline & 14 & 9 & Casa propia & 126 & 84 \\
\hline & 9 & 6 & Casa de hijos & 15 & 10 \\
\hline & 1 & 1 & Otros & 9 & 6 \\
\hline$m=73.61$ & & $=6.37$ & & & \\
\hline
\end{tabular}

\section{Instrumentos}

- Cuestionario de datos sociodemográficos y de perfil jubilatorio. Se elaboró un cuestionario "ad hoc" de 12 preguntas abiertas y cerradas con alternativas fijas, que recaban información sobre características sociodemográficas (sexo, edad, 
nacionalidad, estado civil, nivel de escolaridad, ocupación previa, vivienda, hijos y religión) y jubilatorias (edad de jubilación, antigüedad como jubilado, cantidad de años trabajados en su ocupación previa).

- Cuestionario de Estado de Salud. Con el fin de indagar acerca de la presencia de trastornos psicofisiológicos asociados a la jubilación, se elaboró un cuestionar "ad hoc" que consta de 5 preguntas cerradas con alternativas fijas. Las mismas evalúan si el sujeto percibió alguna modificación en su salud relacionada con su jubilación $\mathrm{y}$, frente a las respuestas afirmativas, se clasifica el tipo de trastorno -cutáneo, urinario, endócrino, osteomusculares, gastrointestinal, cardiovascular, respiratorio, sistema inmune, y del aparato reproductor- (Ortego, López, Alvarez, \& Aparicio, 2012), el momento de aparición y la existencia de antecedentes vinculados al desarrollo del trastorno.

- Índice de Bienestar Psicológico. Esta técnica fue seleccionada con el propósito de estimar la evaluación del senescente acerca de su vida y de sí mismo, en general. El Índice fue estructurado por Stefani (2002), utilizando la forma A del Índice de Satisfacción Vital de Neugarten, Havighurst y Tobin (1961), versión adaptada al medio local por Muchinik (1984). El mismo consta de 11 ítems que definen claramente tres de las cinco dimensiones propuestas por Neugarten: "entusiasmo por la vida", "congruencia entre logros y aspiraciones" y "tono emocional optimista". Asimismo, fue incluida la versión para adultos mayores de Bachman y O'Malley - RSE-B - (1977) de la Escala de Autoestima de Rosenberg (1965), que consta de 10 ítems.

Así, el Índice de Bienestar Psicológico quedó conformado por 21 ítems: 13 afirmaciones directas -direccionadas positivamente- (ítems 1, 3, 5, 6, 8, $9,10,12,13,14,16,18$ y 20$)$ y 8 invertidas -direccionadas negativamente- (ítems $2,4,7,11,15$, 17, 19 y 21). En la administración del cuestionario se le solicita a los sujetos que valoren los mismos conforme a su nivel de acuerdo, a través de una escala de tipo Likert de 3 opciones: "desacuerdo", "indeciso" y "acuerdo". En el caso de las afirmaciones positivas se le asigna un valor de 2 al "acuerdo", 1 al "indeciso" y 0 al "desacuerdo". En el caso de las afirmaciones negativas, se les asigna un valor inverso: 0 al "acuerdo", 1 al "indeciso" y 2 al "desacuerdo".
El cálculo del puntaje total, así como el de las subescalas (Índice de Satisfacción Vital y Escala de Autoestima), se obtiene promediando los valores asignados a cada respuesta. De esta forma se ubica al sujeto en un continuo 0-2, donde un bajo puntaje indica un mínimo nivel de bienestar psicológico, baja satisfacción vital o autoestima negativa, respectivamente, y un alto puntaje señala un alto nivel de bienestar psicológico, alta satisfacción vital o autoestima positiva. Este instrumento fue adaptado al medio local, obteniéndose resultados que indican que posee características psicométricas adecuadas para este tipo de estudio: Alpha de Cronbach $=$ 0.75 (Stefani, 2002) y fue además seleccionado por resultar de fácil administración y evaluación.

\section{Procedimiento}

Se llevaron a cabo las gestiones pertinentes para obtener la autorización para entrevistar a los adultos mayores que asistían a centros de día y talleres dependientes del Gobierno de la Ciudad Autónoma de Buenos Aires y zonas de influencia. Con tal fin, se hizo llegar una copia del proyecto de investigación junto con el consentimiento informado a las autoridades de dichas instituciones. Una vez obtenidos los permisos correspondientes, el protocolo de evaluación fue administrado a los adultos mayores a través de una entrevista individual de una duración aproximada de 40 minutos.

\section{Análisis estadísticos}

Para realizar la descripción de las variables de estudio, se efectuaron cálculos de porcentajes, media aritmética y desviación estándar, de acuerdo al nivel de medición de las mismas.

De acuerdo a la presencia/ausencia de trastornos psicofisiológicos asociados a la jubilación se comparó: a) el puntaje promedio total del Índice de Bienestar Psicológico y sus componentes (Satisfacción Vital y Autoestima) a través del cálculo de media aritmética, desvío estándar y prueba " $\mathrm{t}$ " de Student, y b) los puntajes otorgados por los sujetos para los ítems que conforman el Índice de Satisfacción Vital y Escala de Autoestima, respectivamente, a través de Análisis Multivariados de Variancia. 


\section{Resultados}

\section{Trastornos Psicofisiológicos}

En la tabla 2 y 3 se presentan las frecuencias y porcentajes de los trastornos psicofisiológicos presentes en la muestra. En ellas se observa que el $59 \%$ de la muestra no informó sobre la presencia de algún trastorno psicofisiológico. Por su parte, aquellos que informaron nuevos trastornos psicofisiológicos asociados a la jubilación, reportan que el 39\% son de tipo "cardiovasculares" y el $27 \%$ "osteomusculares", habiendo acaecido en un $82 \%$ "durante el primer año" posterior a haber dejado de trabajar por la jubilación y, en un $71 \%$, sin poseer antecedentes de salud.

Tabla 2.

Presencia de trastornos psicofisiológicos en la muestra $(n=150)$

\begin{tabular}{ccc}
\hline \multirow{2}{*}{$\begin{array}{c}\text { Presencia de } \\
\text { trastornos } \\
\text { psicofisiológicos }\end{array}$} & \multicolumn{2}{c}{$\begin{array}{c}\text { Total } \\
(\mathrm{n}=150)\end{array}$} \\
\cline { 2 - 3 } & $\mathrm{n}$ & $\%$ \\
\hline No & 88 & 59 \\
Sí & 62 & 41 \\
\hline
\end{tabular}

Tabla 3.

Descripción de los trastornos psicofisiológicos $(n=62)$

\begin{tabular}{rcccccc}
\hline $\mathbf{x}$ & $\mathbf{n}$ & $\%$ & $\mathbf{x}$ & $\mathbf{n}$ & $\%$ \\
\hline Tipo de trastorno & & & Momento de aparición & & \\
Cutáneos & 2 & 3 & Durante el primer año & 51 & 82 \\
Endócrinos & 1 & 1 & Después del primer año & 11 & 18 \\
Osteomusculares & 16 & 27 & & & \\
Gastrointestinales & 11 & 18 & No & 44 & 71 \\
Cardiovasculares & 24 & 39 & Antecedentes enfermedad & & \\
Respiratorios & 1 & 1 & Si & 18 & 29 \\
Inmunológicos & 2 & 3 & & & \\
\hline Del aparato reproductor & 5 & 8 & & & &
\end{tabular}

\section{Bienestar psicológico}

Se realizó el cálculo de las medias aritméticas y desvíos estándares para la muestra de los puntajes totales obtenidos en el Índice de Bienestar Psicológico y en las subescalas que lo conforman. La muestra total presentó un puntaje promedio de bienestar psicológico $(m=1.52 ; d e=.32)$, que se refleja en la satisfacción vital $(m=1.33 ; d e=.39)$ y la autoestima positiva $(m=1.73 ; d e=.33)$, denotando una tendencia favorable.

\section{Bienestar psicológico según trastornos psicofi- siológicos}

En la tabla 4 se presentan las medias y desvíos estándares de los puntajes totales del Índice de Bienestar Psicológico para aquellos que manifestaron ausencia y presencia de trastornos psicofisiológicos, así como los resultados de la Prueba t de Student para comparar ambos grupos. Se observa en términos globales (considerando todos los ítems del índice con sus respectivas subescalas) una diferencia estadísticamente significativa para el bienestar psicológico $\left(t_{(148)}=3.462 ; p=.001\right)$, indicando que aquellos que no desarrollaron trastornos psicofisiológicos presentan, con mayor probabilidad, puntajes más altos de bienestar psicológico que aquellos que enfermaron $\left(m_{1}=1.60 d e_{1}=.30 \mathrm{vs}\right.$. $\left.m_{2}=1.42 \mathrm{de}_{2}=.34\right)$.

Tabla 4.

Bienestar psicológico y sus componentes, según presencia de trastornos psicofisiológicos

\begin{tabular}{|c|c|c|c|c|c|}
\hline \multirow{3}{*}{$\mathbf{X}$} & \multicolumn{4}{|c|}{$\begin{array}{c}\text { Trastornos } \\
\text { psicofisiológicos }\end{array}$} & \multirow{3}{*}{$\begin{array}{c}\text { Prueba t de } \\
\text { Student } \\
\mathbf{t}_{(148)}\end{array}$} \\
\hline & \multicolumn{2}{|c|}{$\begin{array}{c}\text { Ausencia } \\
(\mathrm{n}=88)\end{array}$} & \multicolumn{2}{|c|}{$\begin{array}{l}\text { Presencia } \\
\quad(n=62)\end{array}$} & \\
\hline & $\mathrm{m}_{1}$ & $\mathrm{de}_{1}$ & $\mathrm{~m}_{2}$ & $\mathrm{de}_{2}$ & \\
\hline Bienestar psicológico ${ }^{1}$ & 1.60 & .30 & 1.42 & .34 & $3.462^{* * *}$ \\
\hline m= media aritmética & $d e=d e$ & no es: & dar & & $\cdots p s .001$ \\
\hline
\end{tabular}

En las tablas 5 y 6 se consignan las medias y desvíos estándar de los puntajes obtenidos en el Índice de Satisfacción Vital y la Escala de Autoestima, respectivamente, para los subgrupos conformados por aquellos que manifestaron ausencia o presencia de trastornos psicofisiológicos. Asimismo, se consignan en cada tabla los resultados del análisis multivariado de la variancia (MANOVA), considerando los ítems que conforman dichos cuestionarios en forma conjunta y cada uno en forma particular.

En lo concerniente al primer análisis que contempla el MANOVA, los resultados indican un patrón significativamente diferente de respuestas en el Índice de Satisfacción Vital y la Escala de Autoestima según ausencia o presencia de trastornos psicofisiológicos (Satisfacción Vital: $\mathrm{T}_{\text {Hott }}=.144$; FHott ${ }_{(11 ; 138)}=1.812 ; p=.05$; Autoestima: $\mathrm{T}_{\text {Hott }}=.255 ;$ FHott $\left._{(10 ; 139)}=3.539 ; p=.000\right)$. 
Tabla 5.

Índice de Satisfacción Vital según presencia de trastornos psicofisiológicos

\begin{tabular}{|c|c|c|c|c|c|}
\hline \multirow{3}{*}{$\begin{array}{c}\text { Índice de Satisfacción Vital } \\
\text { Ítems }\end{array}$} & \multicolumn{4}{|c|}{$\begin{array}{c}\text { Trastornos } \\
\text { psicofisiológicos }\end{array}$} & \multirow{3}{*}{$\begin{array}{c}\text { MANOVA } \\
T_{\text {Hott }}=.144 \\
\text { FHott }_{(11 ; 138)}=1.812 * \\
F_{(1,148)}\end{array}$} \\
\hline & \multicolumn{2}{|c|}{$\begin{array}{c}\text { Ausencia } \\
\left(n_{1}=88\right) \\
\end{array}$} & \multicolumn{2}{|c|}{$\begin{array}{c}\text { Presencia } \\
\left(\mathrm{n}_{2}=62\right)\end{array}$} & \\
\hline & $\mathrm{m}_{1}$ & $\mathrm{de}_{1}$ & $\mathrm{~m}_{2}$ & $\mathrm{de}_{2}$ & \\
\hline 1. Soy tan feliz como cuando era joven. & 1.26 & .89 & .97 & .87 & $4.038^{*}$ \\
\hline 2. Mi vida podría ser más feliz de lo que es ahora. ${ }^{2}$ & 1.00 & .80 & 69 & .72 & 5.799 * \\
\hline 3. Estos son los mejores años de mi vida. & 1.11 & .81 & .85 & .72 & $4.071 *$ \\
\hline 4. La mayoría de las cosas que hago son aburridas o monótonas. ${ }^{2}$ & 1.53 & .71 & 1.19 & .83 & $7.295^{\star \star}$ \\
\hline 5. Espero para el futuro que me sucedan cosas interesantes y placenteras. & 1.69 & 63 & 1.76 & .47 & .471 \\
\hline 6. Las cosas que hago son tan importantes para mi como siempre lo fueron. & 1.76 & .53 & 1.56 & 64 & 4.237 * \\
\hline 7. Me siento viejo y un poco cansado. ${ }^{2}$ & 1.33 & .81 & .85 & .85 & $11.995^{* * *}$ \\
\hline 8. Al recordar lo que he vivido, me siento muy satisfecho. & 1.69 & .59 & 1.55 & .65 & 2.013 \\
\hline 9. No cambiaría mi vida pasada aunque pudiera hacerlo. & 1.33 & .80 & 1.27 & .75 & .184 \\
\hline 10. Obtuve mucho más de lo que esperaba en la vida. & 1.39 & .73 & 1.29 & .73 & .624 \\
\hline 11. Yo recibí más golpes en la vida que la mayoría de gente que conozco. ${ }^{2}$ & 1.44 & .74 & 1.40 & .74 & .107 \\
\hline $\mathrm{m}=$ media aritmética $\quad \mathrm{de}=$ desvío estándar & & & & & $* * * \mathrm{p} \leq .001$ \\
\hline $\begin{array}{l}{ }^{2} \text { La puntuación de estos ítems fue invertida. Por lo tanto, en todos los ítems q } \\
\text { instrumento, mayores puntajes indican mayores niveles de satisfacción vital. }\end{array}$ & confo & man el & & & $\begin{array}{l}* * \mathrm{p} \leq .01 \\
* \mathrm{p} \leq .05\end{array}$ \\
\hline
\end{tabular}

Tabla 6.

Escala de Autoestima según presencia de trastornos psicofisiológicos

\begin{tabular}{|c|c|c|c|c|c|}
\hline \multirow{3}{*}{$\begin{array}{c}\text { Escala de Autoestima } \\
\text { Ítems }\end{array}$} & \multicolumn{4}{|c|}{$\begin{array}{l}\text { Trastornos } \\
\text { psicofisiológicos }\end{array}$} & \multirow{3}{*}{$\begin{array}{c}\text { MANOVA } \\
\mathrm{T}_{\text {Hott }}=.255 \\
\mathrm{FHott}_{(10,139)}=3.539 * * * \\
\mathrm{~F}_{(1,148)}\end{array}$} \\
\hline & \multicolumn{2}{|c|}{$\begin{array}{c}\text { Ausencia } \\
\quad\left(n_{1}=88\right)\end{array}$} & \multicolumn{2}{|c|}{$\begin{array}{c}\text { Presencia } \\
\quad\left(\mathrm{n}_{2}=62\right)\end{array}$} & \\
\hline & $m_{1}$ & de 1 & $\mathrm{~m}_{2}$ & $\mathrm{de}_{2}$ & \\
\hline 12. Soy una persona digna de estima, al menos en la misma medida que los demás. & 1.87 & .33 & 1.79 & .45 & 1.763 \\
\hline 13. Creo que tengo varias cualidades positivas. & 1.93 & .25 & 1.63 & .55 & $20.514 * * *$ \\
\hline 14. Puedo hacer las cosas tan bien como la mayoría de las personas de mi edad. & 1.84 & .43 & 1.52 & 67 & $13.127^{* \star \star}$ \\
\hline 15. Tengo muy pocos motivos para sentirme orgulloso de mi mismo. ${ }^{3}$ & 1.64 & .57 & 1.39 & .75 & $5.303 *$ \\
\hline 16. Mi actitud frente a mi mismo es positiva. & 1.73 & .50 & 1.50 & 65 & $5.922 *$ \\
\hline 17. Creo que no soy bueno para nada. ${ }^{3}$ & 1.76 & .547 & 1.74 & .51 & .049 \\
\hline 18. Soy una persona útil y por lo tanto, la gente puede contar conmigo. & 1.86 & .345 & 1.85 & .36 & .023 \\
\hline 19. No soy capaz de hacer nada bien. ${ }^{3}$ & 1.84 & .426 & 1.79 & .55 & .405 \\
\hline 20. Cuando hago un trabajo, lo hago bien. & 1.86 & .345 & 1.71 & .52 & $4.702^{*}$ \\
\hline 21. Creo que mi vida no es muy útil. ${ }^{3}$ & 1.65 & .57 & 1.44 & .64 & 4.548 * \\
\hline $\mathrm{m}=$ media aritmética $\quad$ de $=$ desvio estándar & & & & & ${ }^{* * *} p \leq .001$ \\
\hline $\begin{array}{l}{ }^{3} \text { La puntuación de estos ítems fue invertida. Por lo tanto, en todos los items que confor } \\
\text { instrumento, mayores puntajes indican mayores niveles de autoestima. }\end{array}$ & rman e & & & & $\begin{array}{l}* * \\
* p \leq .01 \\
* p\end{array}$ \\
\hline
\end{tabular}

De esta forma, dicho análisis de MANOVA confirma los resultados obtenidos previamente respecto al índice de Bienestar Psicológico, de forma que aquellos entrevistados que manifestaron no haber desarrollado trastornos psicofisiológicos asociados a la jubilación, consignaron mayores puntajes de satisfacción vital y autoestima.
Al considerar cada ítem de forma separada, los valores " $F$ " resultantes del MANOVA muestran un patrón donde las medias del grupo de ausencia de trastornos psicofisiológicos son generalmente mayores al grupo de presencia de trastornos psicofisiológicos. Asimismo, se observan diferencias estadísticamente significativas en algunos ítems del Índice de Satisfacción Vital (tabla 5: en 6 de los 
11 ítems) y de la Escala de Autoestima (tabla 6: en 6 de los 10 ítems), que explicarían las diferencias globales descriptas anteriormente.

Tal como se observa en la tabla 5, aquellos adultos mayores que reportaron ausencia de trastornos psicofisiológicos, en comparación con quienes manifestaron presencia, tienden a presentar puntajes más altos de satisfacción vital, expresando, en mayor medida, no sentirse tan viejos y cansados ( $m_{1}=1.33$ vs. $\left.m_{2}=.85\right)$, no sentir que lo que hacen es tan aburrido o monótono $\left(m_{1}=1.53\right.$ vs. $m_{2}=1.19$ ), sentirse tan felices como cuando eran jóvenes ( $m_{1}=1.26$ vs. $\left.m_{2}=.97\right)$, no sentir que su vida podría ser más feliz de lo que es ahora $\left(m_{1}=1.00\right.$ vs. $\left.m_{2}=.69\right)$, sentir que estos son los mejores años de su vida $\left(m_{1}=1.11\right.$ vs. $\left.m_{2}=.85\right)$ y sentir que las cosas que hace son tan importantes como siempre lo fueron $\left(m_{1}=1.76\right.$ vs. $\left.m_{2}=1.56\right)$.

Asimismo, la tabla 6 indica que aquellos adultos mayores que no desarrollaron trastornos psicofisiológicos, en comparación con quienes sí lo hicieron, tienden a obtener, de forma general, puntajes más altos de autoestima, indicando creer más que: poseen varias cualidades positivas $\left(m_{1}=1.93\right.$ vs. $\left.m_{2}=1.63\right)$, hacen las cosas tan bien como la mayoría de las personas de su edad $\left(m_{1}=1.84\right.$ vs. $m_{2}=1.52$ ), tienen motivos para sentirse orgullosos de sí mismos $\left(m_{1}=1.64\right.$ vs. $\left.m_{2}=1.39\right)$, manifiestan una actitud más positiva frente a sí mismos $\left(m_{1}=1.73\right.$ vs. $\left.m_{2}=1.50\right)$, hacen bien un trabajo al realizarlo $\left(m_{1}=1.86\right.$ vs. $\left.m_{2}=1.71\right) \mathrm{y}$, por último, su vida es muy útil $\left(m_{1}=1.65\right.$ vs. $\left.m_{2}=1.44\right)$.

\section{Discusión}

Los datos recabados en el presente estudio apoyan la hipótesis planteada en cuanto a que quienes informan una menor presencia de trastornos psicofisiológicos asociados a la jubilación tendrían un mayor bienestar psicológico. Asimismo, las relaciones observadas entre satisfacción vital y autoestima, y el desarrollo de enfermedades, coinciden con investigaciones previas. En ellas se encontró que la menor presencia de malestar físico se asociaba a una mayor satisfacción vital (Castellano Fuentes, 2014) y autoestima (Sánchez, Aparicio, \& Dresh, 2006).

En lo referente a los resultados obtenidos sobre el bienestar psicológico para la muestra total, se encontraron niveles altos de satisfacción vital y autoestima positiva. Al respecto, en investigaciones anteriores realizadas sobre muestras con similares características sociodemográficas, se observó que el estar casado y los altos niveles de escolaridad, favorecen el aumento de autoestima (De León Ricardi \& García Méndez, 2016) e influyen sobre los niveles de satisfacción vital (Zavala, Vidal, Castro, Quiroga, \& Klassen, 2006). Al respecto trabajos previos encontraron que las redes de apoyo social y los altos niveles educativos favorecen el bienestar de los adultos mayores (Hertzog, Kramer, Wilson, \& Lindenberger, 2009; Strout \& Howard, 2012).

Por otra parte, si bien la mayor parte de la muestra manifestó no haber desarrollado trastornos psicofisiológicos asociados a la jubilación, quienes sí lo hicieron expresaron no poseer antecedentes y haber enfermado durante el primer año posterior a la jubilación. Estos hallazgos destacan la relevancia de la jubilación como un suceso vital estresante sobre la salud de los adultos mayores, y coinciden con la investigación de Behncke (2012) en la que se observó que el retiro laboral aumenta la probabilidad de contraer diversas enfermedades.

En conclusión, los hallazgos del presente estudio generan evidencia empírica acerca del papel del bienestar psicológico como un aspecto central en la calidad de vida del senescente. Tal como destaca la Organización Mundial de la Salud (2002), existen diversos factores psicosociales de protección de la salud en el adulto mayor, los cuales deben ser considerados al momento de diseñar políticas públicas y nuevas líneas de investigación. Así, el bienestar psicológico resultaría una variable predictora de la salud y protectora frente a los efectos negativos del envejecimiento (Meléndez, Navarro, Oliver, \& Tomás, 2009; Ortiz \& Castro, 2009; Ostir, Markides, Black, \& Goodwin, 2000; Vecina, 2006). $\mathrm{Al}$ respecto, se destaca la importancia de generar programas de preparación para la jubilación que amortigüen el impacto de dicho suceso y las consecuencias que pudiera tener sobre la salud de las personas, favoreciendo el incremento del bienestar $\mathrm{y}$, consecuentemente, promoviendo un envejecimiento saludable (Hernández Triana, 2014; Limón Mendizábal, 1993).

Finalmente se señala que este estudio presenta limitaciones, entre ellas, la imposibilidad de generalizar los resultados por el tipo de muestreo realizado, además de las limitantes propias de un acercamiento de estas características. A todo lo antedicho se suma que la población estudiada pertenece a un nivel socioeconómico medio, destacando la importancia de poder a futuro incluir diferentes niveles socioeconómicos y educativos, cuidando la representatividad poblacional. 


\section{Referencias}

Bachman, J.G. \& O’Malley, P.M. (1977). Self-esteem in young men: a longitudinal analysis of the impact of educational and occupational attainment. Journal of Personality and Social Psychology, 35, 365-380. doi: http://dx.doi. org/10.1037/0022-3514.35.6.365

Baltes, P.B, Reese, H.W. \& Lipsitt, L.P. (1980). Lifespan developmental psychology. Annual Review of Psychology, 31, 65-110.

Behncke, S. (2012). Does retirement trigger ill health. Health Econ., 21, 282-300. doi: http://dx.doi.org/10.1002/ hec. 1712

Biancotti, C., Katz, M., Macotinsky, G., Malvicini, C., Mingorance, D., Paradelo, C. \& Vazzano, L. (2001). Exclusión laboral y reorganización psíquica en el adulto mayor. Cuadernos de Gerontología: Asociación Gerontológica de Buenos Aires (AGEBA), 12(13), 69-73.

Birren, J. (1996). Encyclopedia of Gerontology: Age, Aging and the Aged. New York: Academic Press.

Cano Vindel, A. \& Miguel Tobal, J.J. (2000, marzo). Emociones Negativas (Ansiedad, Depresión e Ira) y salud. Conferencia presentada en el I Congreso Virtual de Psiquiatría.

Casado Morales, M.I. (1996). Psicología y salud. Emociones y enfermedad: una relación compleja e indisoluble. Boletín de la Sociedad Española para el estudio de la ansiedad y el estrés, 5. Recuperado de http://www.ucm. es/info/seas/Boletine/boseas05.htm.

Castellano Fuentes, C.L. (2014). Análisis de la relación entre las actitudes hacia la vejez y el envejecimiento y los índices de bienestar en una muestra de personas mayores. Revista Española de Geriatría y Gerontología, 49(3), 108-114. doi: http://doi.org/10.1016/j.regg.2013.06.001

Castillo-Carniglia, A., Albala, C., Dangour, A.D. \& Uauy, R. (2012). Factores asociados a satisfacción vital en una cohorte de adultos mayores de Santiago, Chie. Gac. Sanit., 26(5), 414-420. doi: http://doi.org/10.1016/j. gaceta.2011.11.021

Cockerham, W.C. (2013). Medical Sociology on the move. Springer Netherlands. doi: https://doi.org/10.1007/97894-007-6193-3.

De León Ricardi, C.A. \& García Méndez, M. (2016). Escala de Rosenberg en Población de Adultos Mayores. Ciencias Psicológicas, 10(2), 119-127. doi: http://doi. org/10.22235/cp.v10i2.1245

Dessors, D. \& Guiho-Bailly, M-P. (Comps.) (1998). Organización del trabajo y salud: de la psicopatología a la psicodinámica del trabajo. Buenos Aires: Lumen/ Hvmanitas

Fernandez-Ballesteros, R. (2007). GeroPsychology. European perspectives for an aging world. Gottingën: Hogrefe.

Figueroa, M. I., Contini, N., Lacunza, A., Levín, M. \& Estévez, A. (2005). Las estrategias de afrontamiento y su relación con el nivel de bienestar psicológico. Un estudio con adolescentes de nivel socioeconómico bajo de Tucumán, Argentina. Anales de Psicología, 21(1), 66-72.

Gatchel, R. J. \& Bianchard, E. (1993). Psychophysiological disorders: Research and clinical applications. Washington: American Psychological Association. doi: http://dx.doi.org/10.1037/10142-000

Hernández Triana, M. (2014). Envejecimiento. Rev Cubana Salud Pública. 40(4), 361-78. Recuperado de http://scieloprueba.sld.cu/scielo.php?script=sci arttext\&pid=S0864-34662014000400011\&lng=es.
Hertzog, C., Kramer, A.F., Wilson, R.S. \& Lindenberger, U. (2009). Enrichment Effects on Adult Cognitive Development: Can the Functional Capacity of Older Adults Be Preserved and Enhanced?. Psych Sci Public Interest, 9(1), 1-65. doi: http://dx.doi.org/10.1111/ j.1539-6053.2009.01034.x

Holmes, T.H. \& Rahe, R.H. (1967). The social readjustment rating scale. Journal of Psychosomatic Research, 11, 213-218. doi: https://doi.org/10.1016/00223999(67)90010-4

Jafri, S.H.R, Ali, F. Mollaeian, A., Hasan, S.M., Hussain, R., Akkanti, B.H., ... El-Osta, H.E. (2017). Major stressful life events and risk of developing lung cancer. Journal of Clinical Oncology 35 (15), 1575. doi: http://dx.doi. org/10.1200/JCO.2017.35.15_suppl.1575

Jiménez, M.G., Izal, M. \& Montorio, I. (2016). Programa para la mejora del bienestar de las personas mayores. Estudio piloto basado en la psicología positiva. Suma Psicológica, 23(1), 51-59. doi: https://doi.org/10.1016/j. sumpsi.2016.03.001

Lawton, M.P. (1991). A Multidimensional View of Quality of Life in Frail Elders. In: J.E. Birren, J. Lubben, J. Rowe, D. Deutchman (Eds)., The Concept and Measurement of Quality of Life (pp. 3-27). New York, Academic Press. doi: https://doi.org/10.1016/b978-0-12-1012755.50005-3

Lazarus, R.S. \& Folkman, S. (1984). Stress, appraisal and coping. New York: Springer.

Limón Mendizábal, M. R. (1993). La preparación a la jubilación: nueva ocupación del tiempo. Revista Complutense de Educación, 4(1), 53-67.

Maddox, G. (1964). Disengagement theory: A critical evaluation. Gerontologist, 4, 80-2. doi: https://doi.org/10.1093/ geront/4.2_Part_1.80

Melendez, J.C., Navarro, E., Oliver, A. \& Tomás, J.M. (2009). La satisfacción vital en los mayores. Factores sociodemográficos. Boletín de Psicología, 95, 29-42.

Montero, I. \& León, O.G. (2002). Sistema de clasificación del método en los informes de investigación en Psicología. Revista Internacional de Psicología Clínica y Salud, 2(3), 115-127.

Mroczek,D.K \& Kolarz, C.M. (1998). The effect of age on positive and negative affect: A developmental perspective on happiness. Journal of Personality and Social Psychology, 75, 1333-1349. doi: https://doi. org/10.1037//0022-3514.75.5.1333

Muchnik, E.G. de (1984). Hacia una nueva imagen de la vejez. Buenos Aires: Editorial de Belgrano.

Neugarten, B. L., Havighurst R. J. \& Tobin, S. S. (1961). The measurement of Life Satisfaction. Journal of Gerontology, 16, 134-143. doi: https://doi.org/10.1093/ geronj/16.2.134

Organización Mundial de la Salud (2002). Definición y evaluación de los riesgos para la salud. En: Informe sobre la Salud en el Mundo (pp. 9-29). Recuperado de http:// www.who.int/whr/2002/en/2002fliersesp.pdf

Ortego M.C., López S., Álvarez M.L. \& Aparicio MM. (2011). Trastornos psicofisiológicos. En: Ciencias Psicosociales II, Universidad de Cantabria. Recuperado de https:// ocw.unican.es/pluginfile.php/1575/course/section/2034/ tema-09.pdf

Ortiz, J., \& Castro, S. (2009). El bienestar psicológico de los adultos mayores y su relación con el autoestima y la autoeficacia. Ciencia y Enfermería, 15(1), 25-31. doi: https://doi.org/10.4067/s0717-95532009000100004 
Ostir, G. V., Markides, K. S., Black, S. A. \& Goodwin, J. S. (2000). Emotional well-being predicts subsequent functional independence and survival. Journal of the American Geriatrics Society, 48, 473-478. doi: https:// doi.org/10.1111/j.1532-5415.2000.tb04991.x

Peiró, J. M. \& Prieto, F. (1996). Tratado de Psicología del Trabajo: Aspectos Psicosociales del Trabajo (vol I \& II). Madrid: Síntesis.

Piqueras Rodríguez, J. A., Ramos Linares, V., Martínez González, A. E. \& Oblitas Guadalupe, L. A. (2009). Emociones negativas y su impacto en la salud mental y física. Suma Psicológica, 16(2), 85-112.

Rojas-Barahona, C.A., Zegers, B., Förster, C.E. (2008). La escala de autoestima de Rosenberg: Validación para Chile en una muestra de jóvenes adultos, adultos y adultos mayores. Rev Med Chile, 137, 791-800.

Rosenberg, M. (1965). Society and the adolescent self image. Princeton: Princeton University Press.

Sánchez M., Aparicio M. \& Dresh V. (2006). Ansiedad, Autoestima y Satisfacción autopercibida como predictores de la Salud, diferencias entre hombre y mujeres. Psicothema, 18(3), 584-590.

Schvarstein, L. \& Leopold, L. (Eds.) (2005): Trabajo y subjetividad. Buenos Aires: Paidós.

Stefani, D. (2000). Prejuicios hacia la vejez: Una forma de violencia social. Revista del Instituto de Investigaciones en Psicología de la Facultad de Psicología, 5(2), 115-124.

Stefani, D. (2002). El efecto de las creencias acerca del sentido de la vida en la participación social del senescente. (Tesis doctoral inédita). Facultad de Medicina, Universidad de Buenos Aires. Argentina.
Strout, K.A. \& Howard, E.P. (2012). The six dimensions of wellness and cognition in aging adults. J Holist Nurs, 30(3),195-204. doi: http://dx.doi. org/10.1177/0898010112440883

Téllez Rivera, V. \& Reyes Montoya, D. (2004). Jubilación, envejecimiento y espacios alternativos de participación social. Rev. Santiago, 104, 102-105.

Vecina, M. L. (2006). Emociones positivas. Papeles del Psicólogo, 1, 9-17.

Villar, F., Triadó, C., Solé Resano, C. \& Osuna, M.J. (2003). Bienestar, adaptación y envejecimiento: cuando la estabilidad significa cambio. Rev Mult Gerontol, 13(3), 152-162.

Wheaton, B. (1996). The domains and boundaires of stress concepts. En H.B. Kaplan (Ed.), Psychosocial stress (pp. 29-70). San Diego, CA: Academic Press.

Zavala, M., Vidal, D., Castro, M., Quiroga, P. \& Klassen, G. (2006). Funcionamiento social del adulto mayor. Ciencia y enfermería, 12(2), 53-62. doi: https://doi. org/10.4067/s0717-95532006000200007

Zegers Prado, B., Rojas-Barahona, C. \& Förster Marín, C. (2009). Validez y confiabilidad del Índice de Satisfacción Vital (LSI-A) de Neugarten, Havighurst \& Tobin en una muestra de adultos mayores en Chile. Terapia Psicológica, 27(1), 15-26. doi: https://doi.org/10.4067/ s0718-48082009000100002 